EPiC Series in Engineering
Volume 3, 2018, Pages 1945-1954
HIC 2018. 13th International
Conference on Hydroinformatics

\title{
Risk Informed Decision-making Framework for Operating Reservoirs Under Flooding Conditions: Accounting for Uncertainty and Risk
}

\author{
Ziad Shawwash $^{1 *}$ and James H. Everett ${ }^{2}$ \\ ${ }^{1}$ Dept. of Civil Engineering, University of British Columbia, Vancouver, Canada \\ ${ }^{2}$ Senior Manager, River Forecast Center, Tennessee Valley Authority, Knoxville, TN, USA \\ shawwash@civil.ubc.ca, jheverett@tva.gov
}

\begin{abstract}
This paper presents the Risk Informed Decision-making Framework and software tool we developed that formally account for flood risk and uncertainty in reservoir operations. The framework and the software tool are intended for practical use by reservoir operations planners to manage flooding events. We present a robust and comprehensive approach that accounts for a multitude of flood risks and their impacts, and that enables its users to identify those alternative reservoir operating plans that formally adopt a state-of-the-art risk informed decision-making framework. Solidly grounded in and closely follows a well-structured planning process, the framework augments existing planning processes and information flows that incorporates specific techniques and methods from probabilistic risk analysis (PRA) and Multi-criteria Decision Analysis techniques (MCDA). Seven major hydropower companies and agencies in North America and Europe sponsored the development of the framework and the decision support tool. We present the results of a case study to illustrate the framework and the software system. We show that numerous advantages can be achieved using such tools over currently used approaches and that in the case of risky and high-impact processes, such as in the management of potentially high-consequence facilities such as storage reservoirs, management by a human operator is essential.
\end{abstract}

\footnotetext{
${ }^{*}$ Corresponding author
} 


\section{Background}

Reservoir operations planners are often confronted with a myriad of conflicting and hard to make decision situations. In particular, managing reservoir and dam facilities during extreme or unusual events can be complex, time consuming and certainly challenging. Most of these events are driven by hydrology, when either too much or too little water is available. Other factors such as unusual operating conditions, dam safety or equipment concerns also drive decision-making. In a typical case, operators try to balance multiple, and at times, competing objectives during these extreme events. In the case of major flooding events, operations planners are usually under time pressure to make decisions when the potential outcomes of different management options are highly uncertain. In such situations, they must quickly make critical and important decisions taking into account the current state of the system and latest available information and forecasts. Their decisions can have significant environmental, social and financial consequences.

Accounting for risk and uncertainty in operating reservoir systems under flooding conditions is a challenging task and requires a well-structured and effective risk informed decision-making framework (National Reserach Council 2015). Such a framework should ensure that operating decisions are made with awareness and assessment of the risks associated with and are aligned with risk policies of the organization and with the values of the stakeholders involved. Such a framework must also support the notion that organizations entrusted with operating reservoir systems should empower and equip their staff with a set of policies and the required tools to help them to formally prepare operating plans that account for uncertainty and risk in making these risky decisions.

This paper presents the results of a CEATI -HOPIG sponsored research project (CEATI 2016) that developed the RIDMF and software tool that can be quickly and efficiently be used by operations planners entrusted to manage reservoir systems during flooding events. We outline the process we have developed to implement the framework for existing reservoir and dam facilities. The framework is solidly grounded in and closely follows a well-structured planning process, and it augments existing planning processes and information flows and incorporates specific techniques and methods from probabilistic risk analysis (PRA) (Kumamoto and Henley 1996) and Multi-criteria Decision Analysis techniques (MCDA) (Keeney and Raiffa 1976). The tool integrates multiple sources of information, it addresses inflow, and impacts uncertainties associated with a major flood event and include trade-off analysis between multiple and competing objectives that are difficult to measure and compare by means of a common unit of measure.

We present the results of one case study to demonstrate the functionality of the framework and the software tool. Results from the case study showed that the framework and the software tool will enable the decisions made in real-time to be less dependent on the operator's own risk tolerance and more aligned with the core values and the corporate risk profile of their organization, and as such can readily be acceptable by senior management and stakeholders involved.

\section{Making the case for RIDMF for reservoir operations under flooding conditions}

RIDM are structured processes to assist decision-makers when faced with high impact, complex decisions involving multiple objectives and with the presence of uncertainty. In operations planning applications, they aim to ensure that decisions between competing operating alternatives are taken with an awareness of the risks associated with each option, and that all attributes of a decision are considered in an integrated manner. The use of these techniques in operations planning of reservoir systems complements but not replace, traditional modelling approaches and human decision makers. 
We are currently witnessing an evolution from risk-based and probabilistic risk analysis approaches to risk-informed decision-making approaches and the realization that quantitative and technical risk assessment approaches can be integrated in the decision-making process when combined with other criteria (e.g., social preferences, political concerns and budget, etc.). This evolution is driven by several factors:

- Better awareness that real risky decisions must integrate multiple concerns;

- Outputs from risk assessment comprise significant uncertainty, and cannot be used "mechanically" to derive a well-founded and risk informed decisions;

- Technical rationality of scientists and engineers are being debated and are seen by some critics to disregard citizens' concerns in the analyses, and this is leading to greater stakeholder participation in decision-making to integrate their concerns, perceptions and values;

- A recognition that both deterministic approaches to technical risk assessment (e.g., safety margins, redundancy and diversity to minimize impacts of failures) and probabilistic methods provide useful insights into safety management, and that a framework for combining these inputs is needed.

Prime examples of such an evolution have been the development and use of RIDMF by (NASA 2010), (IAEA 2011), and (FERC 2014).

\section{The RIDM process}

In an attempt to formalize risk management processes, the International Standards Organization (ISO) issued the ISO 31000 - Risk Management - Guidelines in 2018 (ISO 2018). The Guidelines has been widely adopted around the world by most of G20 countries and other countries. The RIDMF we developed follows ISO 31000 and practically implements its principles, framework and processes for management of reservoir systems under flooding conditions and it consists of three main parts (NASA 2010) and (U.S. Army Corps of Engineers 2009):

- Part 1 - Identification of the decision objectives: understand stakeholders' expectation; derive objectives and performance measures by constructing an objective hierarchy and by deriving performance measures for performance on the objectives; and compile a set of alternatives,

- Part 2 - Risk analysis of decision alternatives: structure the (alternative specific) risk analysis framework; risk quantification via probabilistic modelling of performance; and produce the technical basis for deliberation,

- Part 3 - Risk-informed alternative selection: develop risk-normalized performance metrics; deliberate, select an alternative and document the decision rationale; identify competing alternatives and generate new ones and repeat analysis if needed; communicate the competing alternatives to the decision-maker; and select a decision alternative and document the decision rationale.

\section{RIDMF for Reservoir Operation Under Flooding Conditions}

RIDMF provides a structured framework and software tool for operators of hydro facilities to use when managing reservoir operations under high inflow events. The RIDMF project was sponsored by seven major hydropower institutions, namely: BC Hydro, British Columbia, Canada; Hydro Quebec, 
Quebec, Canada; Sacramento Municipal Utility District, California, USA; Tennessee Valley Authority, Tennessee, USA; Trans Alta, Alberta, Canada; US Bureau of Reclamation, USA; and Vattenfall, Stockholm, Sweden.

The purpose of the RIDMF is to implement a generic framework for the management of processes whose evolution is slow enough to be monitored and influenced by human decisions and actions. The goal of the framework, essentially, is the management of these situations that can evolve into serious incident in which intervention of a human decision-maker is both necessary and essential. A number of interlinked processes some of which rely on inputs prepared well in advance for each reservoir project while others rely on inputs that must be prepared during the flooding event. The conceptual model, methodology and the steps are concerned with a closed feedback loop similar to that illustrated in Figure 1 involving real-time monitoring, validation, simulation/optimization, prediction of likely consequences, quantitative risk assessment, evolution of alternatives, communication with stakeholders, recommendation/ implementation of decisions and documentation of all information used in the decision making process.

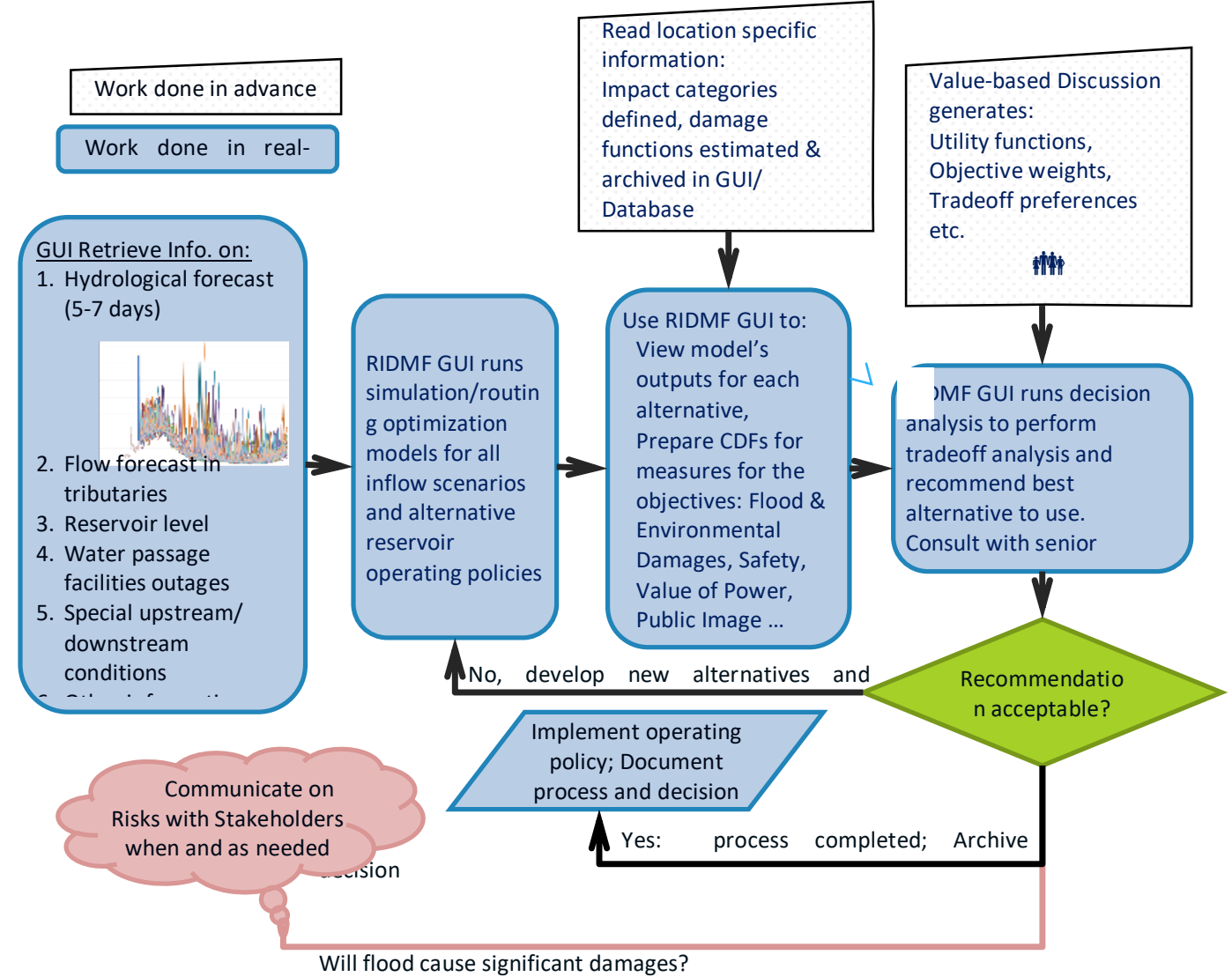

Figure 1: Information flow of the Decision Making Process with RIDMF (CEATI 2016)

\section{Methodology}

Several multi-criteria decision analysis (MCDA) methods exist, including the Simple Additive Weighted method, Analytical Hierarchy Approach, Analytic Network Process, and Multi Attribute Utility Function (Keeney and Raiffa 1976). Among these, the most commonly used methods are the 
Analytic Network Process and the Multi-Attribute Value Function approach. In this work, we have used the Multi-Attribute Value Function approach and assumed that the decision maker is risk-averse with linear utility or discretized utility.

In the Multi-Attribute Value Function approach a utility is an aggregate measure of the relative decision maker's satisfaction with modelled decision outcomes given the objectives. It transforms the measure into metrics that ranges from 0 (worst) to 1 (best).

Three basic utility functions are typically used to capture decision maker's attitude towards risk for each goal as shown in Figure 2:

- Risk Neutral;

- Risk Averse (decreasing slope); and

- Risk Seeking (increasing slope).

- A Combined risk attitude can also be used (e.g., Figure 2).

The multi-attribute utility score for an alternative can be mathematically expressed as follows (U.S. Army Corps of Engineers 2009):

$$
U=\sum_{i=1}^{N} w_{i} V\left(m_{i}\right)
$$



Figure 2: Types of Decision Maker`s Attitude Towards Risk

where, $U$ is the multi-attribute utility score, $w_{i}$ is the preference weight on the objective, $V\left(m_{i}\right)$ is the risk-neutral value score, $m$ is the metric for the objective and $i$ is the index on $N$ objectives.

The RIDMF software system can be configured to run simulation/optimization or reservoir routing models for probabilistic inflow scenarios and alternative reservoir operating policies (see Figure 1). The RIDMF software tool then prepares the cumulative probability distribution functions (CDFs) to describe the performance of the alternatives on the objectives, or $\mathrm{V}(\mathrm{mi})$. Then it runs the decision analysis software module (e.g., (Logical Decisions 2015)) to perform trade-off analysis, rank the alternatives and recommend best alternative to use.

\section{Case Study}

The Pickwick Dam is one of the main hydroelectric facilities in the Tennessee Valley Authority (TVA) system. TVA is a corporate agency of the United States government. The Pickwick Reservoir is the second to last dam on main-stem of the Tennessee River with moderate seasonal storage and large drainage area of about 33,000 square miles above the dam. The average annual flow amounts to 53,900 cubic feet per second (cfs) and the maximum known flood occurred in March 1973 was estimated at 585,400 cfs (CEATI 2016).

The Pickwick is a multi-purpose reservoir and is operated to satisfy multiple objectives: flood damage reduction; commercial navigation; hydroelectric power generation; water supply for different purposes including municipal for local communities, irrigation, industrial (e.g., paper industry) and environmental flows; water quality; recreation use; and dam safety.

\subsection{Case Study}

The overall goal is to develop the best operation for high inflow events. 


\subsection{Operating Alternatives}

Three operating alternatives to operate the reservoir for this high inflow event were investigated:

- Normal Dam Operation, that follows operations procedures typically used by load factoring the system to meet system power needs and to maximize energy production opportunities;

- Maximizing Hydro Generation, to maximize power output by running power generating facilities at their maximum output;

- Maximize Flood Storage Recovery, to release pre-specified amount of water to evacuate enough storage capacity to reduce the peak reservoir outflows.

lists the objectives, their measures, units of measurement, and weights as used in this study.

\subsection{Operating Alternatives}

Three operating alternatives to operate the reservoir for this high inflow event were investigated:

- Normal Dam Operation, that follows operations procedures typically used by load factoring the system to meet system power needs and to maximize energy production opportunities;

- Maximizing Hydro Generation, to maximize power output by running power generating facilities at their maximum output;

- Maximize Flood Storage Recovery, to release pre-specified amount of water to evacuate enough storage capacity to reduce the peak reservoir outflows.

\begin{tabular}{llc}
\hline \multicolumn{1}{c}{ Objectives, $i$} & \multicolumn{1}{c}{$\begin{array}{c}\text { Measures, } m \\
\text { (Units) }\end{array}$} & $\begin{array}{c}\text { Objective } \\
\text { Function } \\
\text { Weight, } w_{i}\end{array}$ \\
\hline Max. Power Generation & \multicolumn{1}{c}{ Energy Generation (GWHr) } & $10 \%$ \\
Min. Impact on Affected Crop Area & Affected Crop Area (Hectares) & $10 \%$ \\
Min. Impacts on Commercial Navigation & Commercial Navigation (Flow, cfs) & $10 \%$ \\
Min. Impacts on Dam Safety & Dam Safety Pool Level (Elevation, ft) & $30 \%$ \\
Min. Structural Flood Damage & Structural Flood Damage (\$) & $40 \%$ \\
\hline
\end{tabular}

Table 1: Operations objectives, measures, performance measurement units and objective weights

\subsection{Pickwick Case Study Results and Discussion}

Several results can be synthesized for the Pickwick case study as discussed below.

\subsubsection{Performance of the alternatives on the objectives}

Figure 3 illustrates the performance of the alternatives on the objectives. Each alternative bar length is proportional to performance on the overall goal as measured by the expected value of the overall goal. It is made up of a stack of bars showing the influence of the various measures on the overall goal. It can be seen, for example, that Maximize Flood Storage Recovery operation performs best on the overall goal of finding the best operating alternative.

It can also be seen that the structural flood damage and the dam safety measures dominates the overall utility for the three alternatives and it seems that in this analysis dam safety differentiates flood storage recovery operation from for other alternatives. Another interesting and useful output of such an analysis consists of uncertainty summaries for the alternatives with respect to a single 
measure or goal as shown in Table 2. These results are derived from a 5000 Monte Carlo simulations for each alternative. Of particular interest, for example, is the variability and sensitivity of the simulated impacts as expressed by the value of standard deviation and other statistics. Columns 1-5 and 8 are self-explanatory while the $5 \% \mathrm{P}$ and $95 \% \mathrm{P}$ columns represent simulation results of the overall goal utility in which only five percent of the sampled trials were below $(5 \% \mathrm{P})$, while the $95 \% \mathrm{P}$ column represents of the overall goal utility in which only five percent of the simulation trials were above. These statistics are also available for each objective in the study.

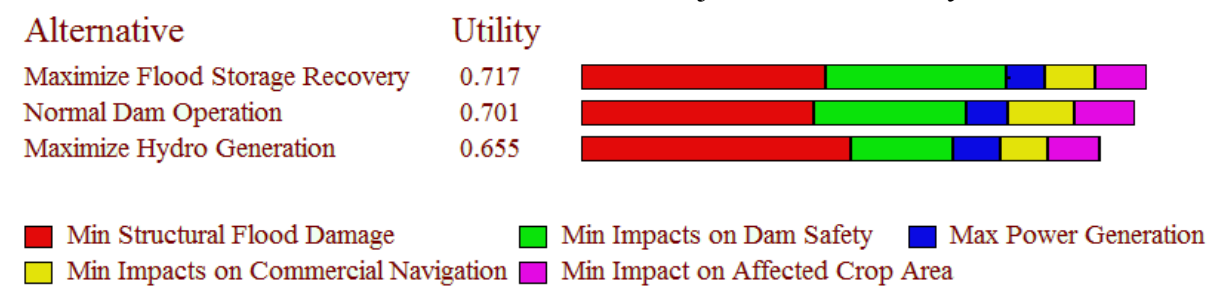

Figure 3: Performance of the operating alternatives on the objectives

\begin{tabular}{lccccccc}
\hline Alternative & Mean & Std. Dev. & Median & Min & $5 \% \mathrm{P}$ & $95 \% \mathrm{P}$ & Max \\
& & & & & & & \\
\hline Max. Flood Storage Recovery & 0.716 & 0.125 & 0.721 & 0.333 & 0.486 & 0.900 & 0.980 \\
Max. Hydro Generation & 0.654 & 0.129 & 0.653 & 0.253 & 0.446 & 0.870 & 0.977 \\
Normal Dam Operation & 0.700 & 0.123 & 0.710 & 0.292 & 0.487 & 0.891 & 0.976 \\
\hline
\end{tabular}

Table 2: Summary of utility uncertainty for best operation for high inflow goal

\subsubsection{Uncertainty in performance of the alternatives on overall goal}

Figure 4 illustrates the sampling results of the decision analysis step and shows non-parametric discrete and cumulative distribution functions for 5000 simulation to assess the performance of the alternatives on the study objectives and overall goal. It can be seen that Max. Flood Storage Recovery operation (Figure 4(b)) performs only slightly better than Normal operations while it performs much better than the Max. Hydro Generation operation as the Max. Storage Recovery tends to steadily increase towards the higher end of the utility (horizontal) axis while those for other alternatives tend to cluster at low and mid utility values.

\subsubsection{Pairwise Utility and Trade-off Functions of Objective Function Measures}

Pairwise utility function contour curves provide a view of the sets of equally preferred levels on the two measures. Figure 5 shows pairwise utility function curves for Dam Safety Pool Level vs Structural Flood Damage (a), Energy Generation (b), Commercial Navigation, and (c) Affected Crop Area (d). Crosses (+) that appear on the graph represent the performance levels of alternatives on the two measures. The graph can also be used indirectly to assess the trade-off between two objectives (e.g., by assessing the steepness, curvature and orientation of the contours). In this analysis the utility functions for all the objectives were assumed to be linear and it can be seen that this have resulted in a linear relationship between the utilities.

Analysis of the trade-offs between the objective functions can be used to develop better understanding of the interactions between the objectives and the trade-offs involved as they give an indication of the expected gain on one objective and the corresponding loss on other objectives. For example, Figure 5 shows the trade-off functions of Dam Safety with the other objectives. It should be noted that such graphs are very useful and effective tools in clarifying the interaction between the objectives in stakeholder negotiation and for decision-making processes in organizations. 
The efficient frontier defines the sets of alternatives that provide the highest ratio of benefits to costs. The efficient frontier is formed by those sets of alternatives that provide the highest total benefit for any given total cost. The sets of alternatives forming the efficient frontier are the sets closest to the top left corner of the graph (e.g., maximum benefits) when objectives are maximized. Figure 6 illustrates the efficient frontier for the Dam Safety objective and it can be seen that the Maximum Flood Storage Recover alternative performs best among the alternatives mainly due to the comparatively low head water reservoir. Many other analysis results can be investigated and explored in such a complex decision-making process.
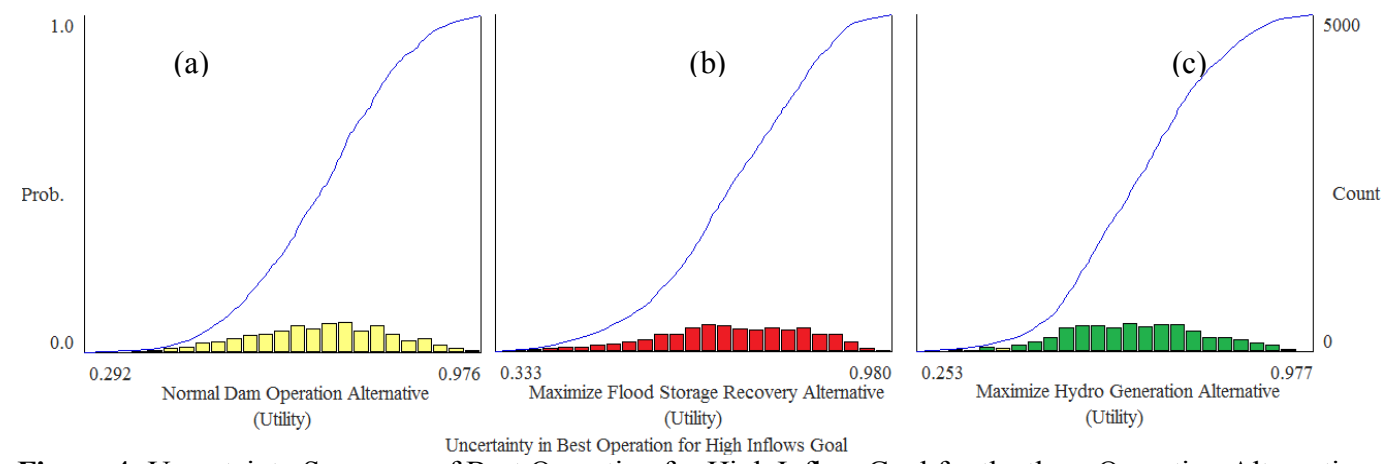

Figure 4: Uncertainty Summary of Best Operation for High Inflow Goal for the three Operating Alternatives
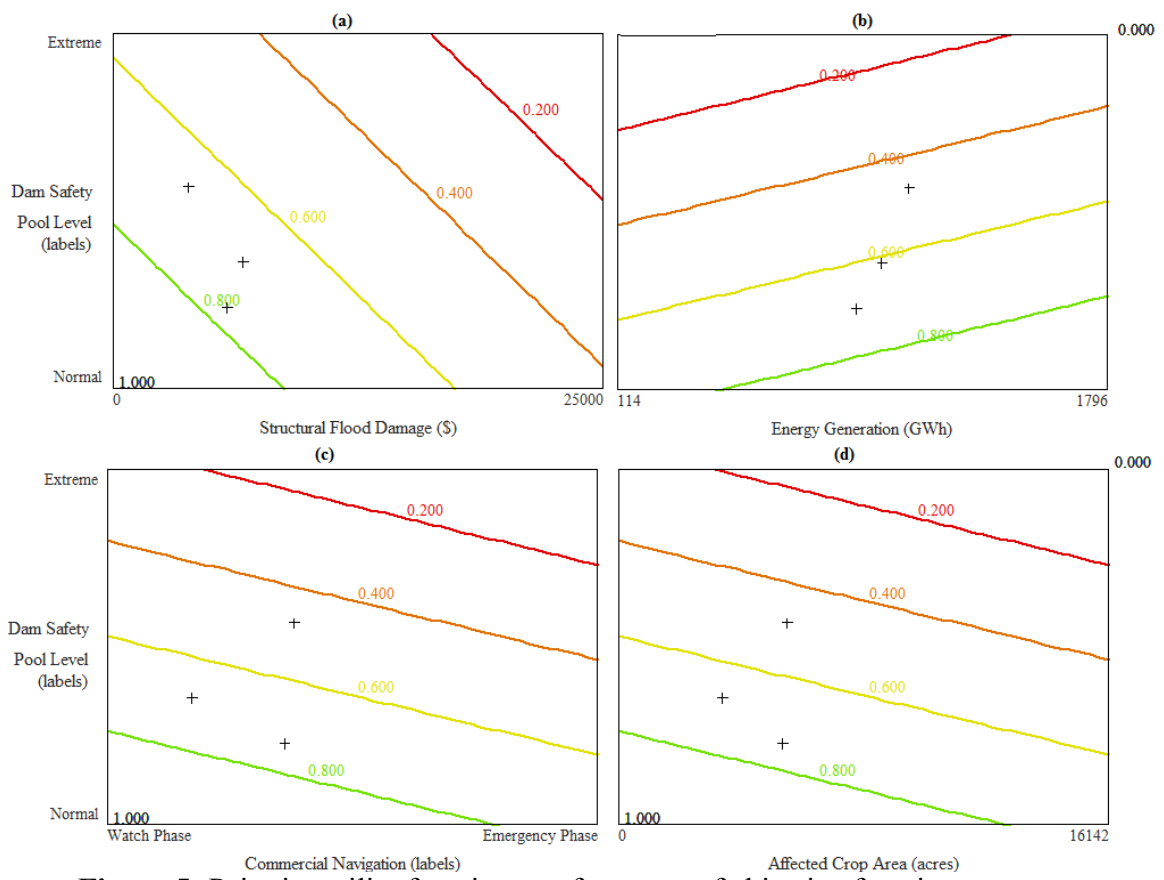

Figure 5: Pairwise utility functions performance of objective function measures 




Figure 6: Efficient Frontier for overall goal vs dam safety pool level objective

\section{Conclusions}

Reservoir facilities represent a major infrastructure investment made by most nations around the world. They are essential for long-term supply of water and clean and renewable energy and in many instances for flood risk management. These storage facilities can, however, pose significant public, economic and environmental and safety concerns particularly during times of high inflow events. Decision makers who regulate and operate these facilities are increasingly adopting risk-informed approaches to help rationalize and support their operating decisions to ensure public safety and to achieve goals and objectives desired by their organizations and stakeholders.

This paper presented the development of an integrated, systems-based practical approach to formally account for risk and uncertainty and to make risk-informed reservoir operating decisions under flooding conditions. The RIDMF is a state-of-the-art framework implemented in a software application to enable the reservoir operations planners to:

- Build and maintain a database of goals/objectives, impact measures and the latest/seasonal preferences and value trade-offs for specific reservoir projects,

- Incorporate in the decision the uncertainty in hydrology and impacts and concerns,

- Quickly and efficiently develop and evaluate operating alternatives by making trade-offs between risks, costs, and other decision outcomes and to perform sensitivity analysis,

- Facilitate communication about uncertainty, risk attitudes, value trade-offs and all data used in decision making and document and archive the state of the systems and the decision made for the event.

The RIDMF prototype can be easily adapted and generalized for use in production for making reservoir operations, scheduling, maintenance, trading decisions and for the analysis of dam safety and general investments decisions. The framework is implemented in a software system that can be easily adapted for any reservoir system and can further be used in the analysis of dam safety decisions and to make operating decisions.

We presented the results of a case study to illustrate the framework and software system to demonstrate the applicability of the framework using an existing hydropower system in Tennessee,

USA. Case study results illustrated some of the functionality of the tool towards identifying alternative operating plans that formally adopt a state-of-the-art risk informed decision making framework. Feedback from the reservoir operators indicated that the recommendations made by the RIDMF system meets their expectations. We expect that users of the framework and the software will achieve numerous advantages over currently used approaches in reservoir operations. 
It can be concluded that in the case of risky and high-impact and high-consequence processes management by a human operator is essential. Therefore, every effort should be made to implement state-of-the-art decision support tools, such as those presented in this paper, to help the human operators of these critical infrastructure system to rapidly assess the situation, determine the consequences of potential decisions, assess and communicate the risks, and make risk informed decisions that are aligned with the risk policies of the organization and with the values of the stakeholders involved.

\section{References}

CEATI. Final Report: Risk Informed Decision Making Framework for Hydro Reservoir Systems with Limited Regulating Capability under High Inflow Conditions: Accounting for Uncertainty and Risk. Vancouver, Canada: Shawwash Consulting Inc., 2016.

FERC. FERC Risk-informed decision making framework: Washington D.C.: FERC, 2014.

IAEA. A Framework for an Integrated Risk Informed Decision Making Process. INSAG-25, , the International Atomic Energy Agency (IAEA). International Nuclear Safety Group, International Atomic Energy Agency (IAEA), Vienna, Austria: International Atomic Energy Agency (IAEA), 2011.

ISO. "ISO Guide 31000 (2018) Risk management — Guidelines." 2018.

Keeney, R., H. Raiffa, and H. Raiffa. Decisions with Multiple Objectives: Preferences and Value Tradeoffs. New York: Wiley, 1976.

Kumamoto, E.J., and H. Henley. Probabilistic risk assessment for engineers and scientists. New York, USA: IEEE Press, 1996.

Logical Decisions. Logical Decisions ${ }^{\circledR}$ for Windows, Version 6.2. Logical Decisions, 2015.

NASA. "NASA Risk-Informed Decision Making Handbook." NASA Headquarters, Washington D.C., USA, 2010.

National Reserach Council. Tying Flood Insurance to Flood Risk for Low-Lying Structures in the Floodplain. N.W. Washington, D.C. 20481: National Academy of Sciences, 2015.

U.S. Army Corps of Engineers. Risk-informed decision framework for Louisiana coastal protection and restoration. New Orleans District, Mississipi Valey Division, USA: U.S. Army Corps of Engineers, 2009.

\section{Biography}

Ziad K. Shawwash holds Ph.D. in Civil Engineering in Hydrotechnical/water resources planning and management from the University of British Columbia in Vancouver, Canada. He is a professor at the Department of Civil Engineering, UBC and his position is fully sponsored by BC Hydro and his research is sponsored by NSERC and BC Hydro and is mainly focused on hydropower system operations planning, wind integration, risk informed decision making for water resources planning and management, and flood risk management. Ziad is also the Director of Shawwash Consulting Inc. (www.shawwash.com). E-mail: shawwash@mail.ubc.ca

James H. Everett is the Senior Manager of River Forecast Center, Tennessee Valley Authority. He holds B.A.Sc. \& M.A.Sc. in Civil and Environmental Engineering from the University of Tennessee in Knoxville, Tennessee. He is also a registered professional engineer in the State of Tennessee. Email: jheverett@tva.gov 